\title{
An EROI-Based Analysis of Renewable Energy Farms with Storage
}

\author{
Y. Ghiassi-Farrokhfal， S. Keshav， C. Rosenberg \\ University of Waterloo \\ \{yghiassi, keshav, cath\}@uwaterloo.ca
}

\begin{abstract}
Large renewable energy (RE) farms, such as wind or solar farms are usually sited in remote areas, far from the transmission grid which typically interconnects population centers. Thus, they need to be connected on expensive access lines (distribution feeders) with limited capacity. The excess of RE generation over line capacity is wasted; this is called curtailment. We study curtailment using the metric of energy return on investment (EROI), defined as the ratio of useful energy extracted from each unit of energy invested in creating the renewable energy generation system. Curtailment reduces EROI. It may appear that we can extract more energy from an RE farm and increase EROI by adding storage to the system, where this storage is charged during generation peaks and discharged during off-peak times. However, manufacturing the storage requires an energy investment, and, after a certain number of cycles of usage, the storage becomes non-functional. Thus, adding storage may actually decrease the EROI. In this work, we study the EROI for RE farms when used with several types of storage technologies. Unlike prior work that makes numerous simplifying assumptions, our work accounts for storage size and storage imperfections and uses actual traces of renewable power generation. We find that lithium-ion batteries increase the EROI of both wind and solar farms, unlike lead-acid batteries which generally decrease their EROI. We also show that increasing access line capacity to achieve a target EROI is much more expensive for solar farms than for wind farms.
\end{abstract}

\section{INTRODUCTION}

Large renewable energy (RE) farms, such as wind farms and solar farms, are usually sited in remote areas [21]. Being far from the transmission network that typically interconnects population centres, they require the provisioning of access lines (distribution feeders). Extracting the entire energy from RE farms requires access lines to support generation peaks, because generation in excess of the line capacity must be curtailed $^{1}$. However, increasing access line capacity is costly; hence, line capacity is typically inadequate to support peak generation, which limits the amount

\footnotetext{
${ }^{1}$ Curtailment may also occur due to lack of sufficient demand. We discuss this in more detail in Section 3.
}

of energy and potential revenue that can be extracted from these farms [21]. This waste of power is undesirable for RE farm owners, who must wait longer to recoup their initial capital investment as a result.

We study curtailment using the metric of energy return on investment (EROI), defined as the ratio of useful energy extracted from each unit of energy invested in creating an RE generation system, such as the energy required to manufacture wind turbines and photovoltaic (PV) panels. Curtailment reduces the EROI of the system because it reduces the amount of energy extracted from the energy system.

One way to increase EROI is to use devices that store surplus power that cannot be carried by an access line and discharge it when there is enough spare capacity. It may appear that all storage technologies invariably increase the EROI of RE farms. However, this does not take into account the energy required to manufacture storage systems, which have limited lifetimes. Recent work has shown that adding an energy-intensive and short-lived storage system to prevent curtailment may actually reduce the overall EROI: it would be better to simply curtail generation than to use such storage devices [6]. While the conclusions in [6] are drawn based on a simple model, our work formulates an accurate EROI analysis for this problem and makes the following contributions:

1. We provide a generic formulation for computing the EROI for a variable-rate RE generation system coupled with storage, and transmitting power over an access line with limited capacity. Our formulation accounts for the storage state of charge and the physical constraints of the storage technologies.

2. We use actual solar and wind measurement datasets to evaluate system performance. We contradict some of the earlier claims in [6]. We find that for both wind and solar power, some storage technologies increase EROI compared to curtailment and some do not.

3. Our formulation enables us to size storage in order to obtain an EROI arbitrarily close to the EROI of the same storage technology with infinite size. Moreover, it also demonstrates that the required access line capacity for a target EROI is much larger for solar power 


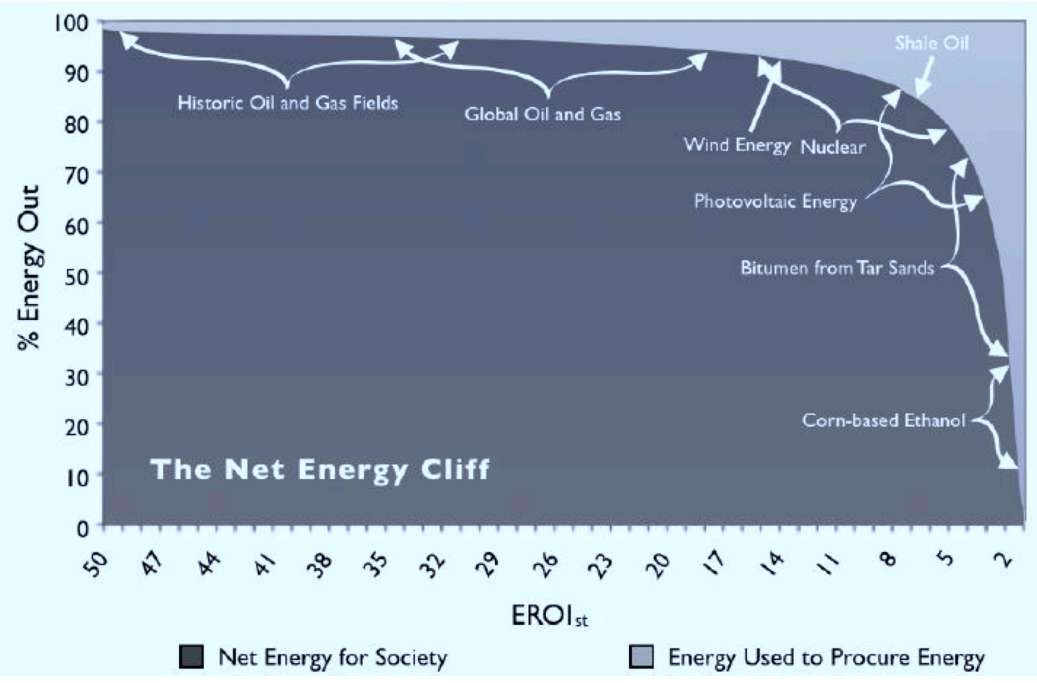

Figure 1: The net energy cliff [18]: The light grey area represents the percent of the total energy return that is invested. The dark grey area is the percentage of the energy returned to the total energy invested and returned (i.e., $100 \times \frac{E R O I}{E R O I+1}$ ). Note that the net energy is very sensitive to EROI values below 8 .

\begin{tabular}{|c||c|}
\hline & EROI \\
\hline Coal & 80 \\
\hline Gas & 10 \\
\hline Nuclear & 9 \\
\hline Wind turbine & 18 \\
\hline Solar PV panel & 9 \\
\hline Biomass & 1.3 \\
\hline
\end{tabular}

Table 1: EROI of some of the power sources [15]

than for wind power, requiring a more expensive integration system for solar power.

The rest of the paper is organized as follows. We motivate the importance of EROI analysis in Section 2. We discuss the challenges of integrating renewables to the grid in Section 3. We introduce our system model and formulate the EROI for that system in Section 4. Numerical examples and engineering insights are presented in Section 5 and we review the related work in Section 6. We conclude the paper and discuss future work in Section 7.

\section{EROI}

Designing and evaluating an energy system is carried out largely by focusing on its cost efficiency. As an example, an $\mathrm{RE}$ farm is typically sized to maximize its revenues while taking investment costs and some requirement constraints into account. Another important factor, though often ignored, is energy efficiency. The energy efficiency of an energy system mimics its cost efficiency. One of the most widely-used metrics to measure the energy efficiency is the energy return of that system during its lifetime per unit energy invested to create that system (EROI) [15]:

$$
E R O I=\frac{\text { Energy returned in }\left[0, T_{s y s}\right]}{\text { Energy invested in }\left[0, T_{s y s}\right]},
$$

where $T_{\text {sys }}$ is the lifetime of the system. When $T_{\text {sys }}$ is not known, or the system consists of many components with different lifetimes, the following equation is alternatively used to compute EROI

$$
E R O I=\lim _{T \rightarrow \infty} \frac{\text { Energy returned in }[0, T]}{\text { Energy invested in }[0, T]} .
$$

One of the main criticisms of EROI is that it does not take time into account. Specifically, for a given investment, EROI shows the total return, but it does not indicate how soon this return will occur.

EROI is widely used in the literature as a metric for the quality of fuels and energy systems (see Table 1); higher quality fuels are those with higher EROIs. Fossil fuels have a large EROI, which ensures reasonable energy return for any energy system, making cost efficiency the main concern. Thus, EROI analysis has been mostly ignored to date; however, it has recently become an important metric for two reasons.

First, an unfavourable decreasing trend has been observed on the EROI of fossil fuels (except coal). For instance, the global EROI for the production of oil and gas has declined from 30 in 1995, to about 18 in 2006. The second reason motivating EROI analysis is the increasing trend towards $\mathrm{RE}$ to reduce carbon use. However, most RE sources have considerably lower EROI values than fossil fuels. Thus, renewables might not be as energy profitable as originally suggested. We believe that the fact that high quality (high EROI) fossil fuels are employed in the creation, transport, and implementation of wind turbine and PV panels needs to be taken into account when performing a viability study of an RE system (along with other aspects such as cost).

When evaluating the EROI of a system, it is important to note that a small change in EROI is far more critical for small values of the EROI than it is for large values. This 


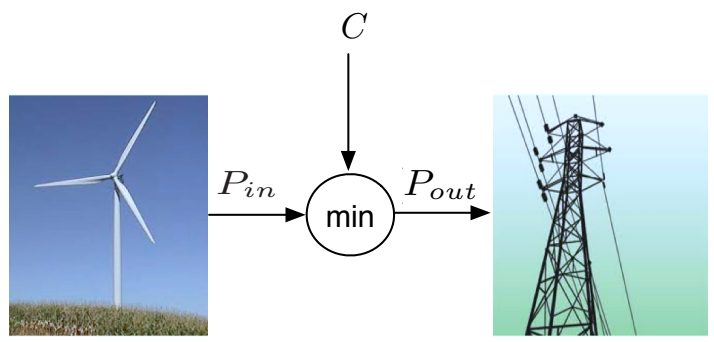

(a) Model of our system

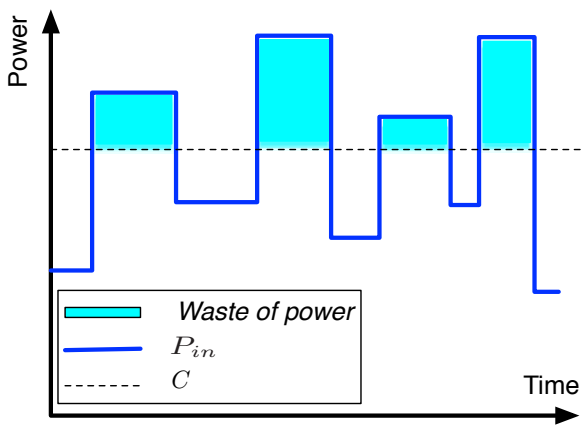

(b) Waste of power due to curtailment

Figure 2: A simple model for curtailment: when a renewable energy source produces more power than can be carried on a transmission line of capacity $C$, the excess is wasted.

becomes apparent from Fig. 1. Here, for a given EROI, the dark grey portion represents the percentage of the energy returned as a fraction of the total energy invested and returned (i.e., $\left.100 \times \frac{E R O I}{(E R O I+1)}\right)$. This is commonly viewed as the energy efficiency of the system [18]. It is clear that the impact of changing EROI on energy efficiency is much less pronounced for large values of EROI. As EROI decreases, we reach an energy cliff, where slightly decreasing EROI considerably reduces the energy efficiency. This observation highlights the fact that when the EROI of a system is small, even a small change in EROI can significantly affect energy efficiency. Also, note that an EROI lower than 8 yields an energy efficiency lower than $90 \%$ and this percentage decreases very steeply with decreasing EROI. We therefore conservatively view an EROI of 8 as the minimum desirable from an RE farm. Although this is a somewhat arbitrary choice, in another work, it has been shown that a minimum EROI value of 3 is needed to support continued economic activity [13].

The EROI of renewables is significantly lower than those of fossil fuels and conventional power sources. Moreover, curtailment further reduces their EROI. Thus, there is an urgent need to improve the EROI of RE technologies. This can be accomplished using three complementary approaches: (1) reducing the energy needed to create RE systems, (2) increasing the lifetime of RE technologies, and (3) reducing $\mathrm{RE}$ curtailment using storage. In the rest of this paper, we focus on the third approach.

\section{INTEGRATING RE FARMS}

To recap, a major challenge facing RE farms is their reduced energy efficiency due to curtailment. Curtailment is necessary for two reasons: insufficient line capacity for generation peaks and insufficient demand. It has been shown that limited line capacity is the most important reason for reduction in EROI today [10] and is therefore the primary focus of our work.

We also do not consider curtailment due to lack of demand because this is an extremely complex problem. The gener- ation mix needed to meet a given load includes must run generators, such as nuclear generators, which are insensitive to demand, and fast spinning generators, such as natural gas turbines or combined hydro generators, which can respond quickly to reduced demand. In the future, moreover, loads may also be met from energy stored in storage devices, that have their own constraints on capacity and ramping rates. Thus, it is difficult to determine the degree to which RE farms must be curtailed due to insufficient load, and we defer consideration of this difficult issue to future work.

In the remainder of this section, we will consider the integration of RE farms in the absence and presence of storage, respectively.

\subsection{Systems without storage}

In a system without storage, the surplus power exceeding the access line capacity is curtailed (Fig. 2a). Curtailment is a good strategy in that it does not require any new investment. Interestingly, it also has the effect of making RE farms more attractive. This is because curtailment allows an RE farm to be deployed while ignoring, to first order, access line constraints, by simply shedding generation when it peaks exceed capacity. However, increasing RE farm capacity beyond a certain threshold wastes too much power and leads to poor energy and cost efficiencies [9], [16], [19].

\subsection{Systems with storage}

An alternative to curtailment is to use storage. By adding a storage device adjacent to a transmission line, we can store the non-absorbable power and withdraw it later. The stored energy can be used later to fill the gap between the available power and the access line capacity.

There are multiple advantages to using storage for renewable energy integration: reducing power waste, price arbitrage, peak shaving, and power regulation, among others [7]. Adding storage to facilitate renewable energy integration, however, comes at an investment cost, financially (the price of storage) and from energy perspective (the energy invested to create the storage device). In this paper, we are 
concerned about the latter investment as we study the energy efficiency of adding storage devices to access lines.

In the next section, we formulate and compare the EROI of the two systems above (with and without storage) to understand whether or not adding storage can improve the EROI of the system.

\section{EROI FORMULATION AND COMPARI- SON}

We assume a discrete-time model, where time is slotted $t=0, T_{u}, 2 T_{u}, \ldots$, with $T_{u}$ being the time unit. To simplify notation, we drop $T_{u}$ from our formulation by assum$\operatorname{ing} T_{u}=1$. Generalizing the formulas for any $T_{u}$ is a matter of additional notations.

We formulate the EROI of the systems without storage and of those using storage, separately, in the following. Please refer to Table 2 for the notation used in the rest of the paper.

\subsection{The EROI of a system without storage}

Let $P_{i n}(t)$ be the available power from an energy harvesting technology, such as a wind or solar farm, at time $t$. Suppose we want to transport $P_{i n}$ over an access line of capacity $C$ (see Fig. 2). Without access to storage in this system, the available power exceeding the access capacity must be curtailed. Hence, the absorbable power, $P_{\text {out }}$ (i.e., the power effectively carried by the access line), in this system at any time $t$, is given by:

$$
P_{\text {out }}(t)=\min \left(P_{\text {in }}(t), C\right) .
$$

Let $E_{\text {in }}(T)$ and $E_{\text {out }}(T)$ be, respectively, the total available energy from the energy harvesting technology and the total absorbable energy by the access line in the time interval $[0, T]$ for some $T>0$. This is:

$$
E_{i n}(T)=\sum_{\tau=0}^{T} P_{i n}(\tau)
$$

and

$$
E_{\text {out }}(T)=\sum_{\tau=0}^{T} P_{\text {out }}(\tau)
$$

A key metric in designing a wind or solar farm is the waste ratio, $R$, defined as:

$$
R=\lim _{T \rightarrow \infty} \frac{\text { Total wasted renewable energy in }[0, T]}{\text { Total available renewable energy in }[0, T]} .
$$

The larger is $R$, the smaller the dispatchable fraction of the total available renewable power. This, subsequently, impacts the time to return the initial capital cost of the system [4]. With current technologies, prices, and energy market

\begin{tabular}{|c|c|c|}
\hline & Name & Description \\
\hline$*$ & $P_{i n}(t)$ & Available renewable power at time $t(\mathrm{~W})$ \\
\hline \multirow[t]{4}{*}{ * } & $P_{\max }$ & Peak generation capacity of the renewable energy farm (W) \\
\hline & $P_{\text {out }}(t)$ & Absorbable power by the access line at time $t(\mathrm{~W})$ \\
\hline & $P_{a d d}(t)$ & Part of the $P_{\text {out }}(t)$ coming from storage $(\mathrm{W})$ \\
\hline & $P_{\text {direct }}(t)$ & Part of the $P_{\text {out }}(t)$ not coming from storage $(\mathrm{W})$ \\
\hline \multirow[t]{5}{*}{ * } & $E_{\text {in }}(T)$ & Total renewable energy in $[0, T](\mathrm{Wh})$ \\
\hline & $E_{s}(T)$ & Total energy output from storage in $[0, T](\mathrm{Wh})$ \\
\hline & $E_{\text {out }}(T)$ & Energy transmitted over the access line in $[0, T](\mathrm{Wh})$ \\
\hline & $E_{a d d}(T)$ & Part of the $E_{\text {out }}(T)$ coming from storage (Wh) \\
\hline & $E_{\text {direct }}(T)$ & Part of the $E_{\text {out }}(T)$ not coming from storage (Wh) \\
\hline \multirow[t]{4}{*}{$*$} & $C$ & Access line capacity (W) \\
\hline & $b(t)$ & State of charge at time $t(\mathrm{Wh})$ \\
\hline & $E R O I_{0}$ & EROI of the curtailment scenario \\
\hline & $E R O I_{B}$ & EROI of the storage scenario \\
\hline$*$ & $E R O I_{e}$ & EROI of the energy harvesting technology \\
\hline$*$ & $E R O I_{s}$ & EROI of the storage device \\
\hline \multirow[t]{4}{*}{$*$} & $\varepsilon_{s}$ & Energy invested to create each unit of energy storage (Wh) \\
\hline & $E_{\text {lifetime }}^{*}$ & Total deliverable energy during storage lifetime $(\mathrm{Wh})$ \\
\hline & $R_{0}$ & Waste of power ratio in a curtailment system \\
\hline & $R_{B}$ & Waste of power ratio in a system with storage \\
\hline$*$ & $B$ & Storage size $(\mathrm{Wh})$ \\
\hline * & $\alpha_{c}\left(\alpha_{d}\right)$ & Storage charging (discharging) power limit (W) \\
\hline * & $\eta$ & Storage efficiency \\
\hline * & $\gamma$ & Storage leakage power rate $(\mathrm{W})$ \\
\hline * & $D o D$ & Storage depth of discharge \\
\hline \multirow[t]{2}{*}{$*$} & $\lambda$ & Storage cycle life \\
\hline & $\lambda_{c r}$ & Critical cycle life \\
\hline
\end{tabular}
schemes, the average waste ratio for wind power ranges from as large as $16 \%$ and as low as $1 \%$ [20].

We denote $R_{0}$, the waste ratio in a no-storage scenario,
Table 2: Notation. Starred variables are input to the analysis.

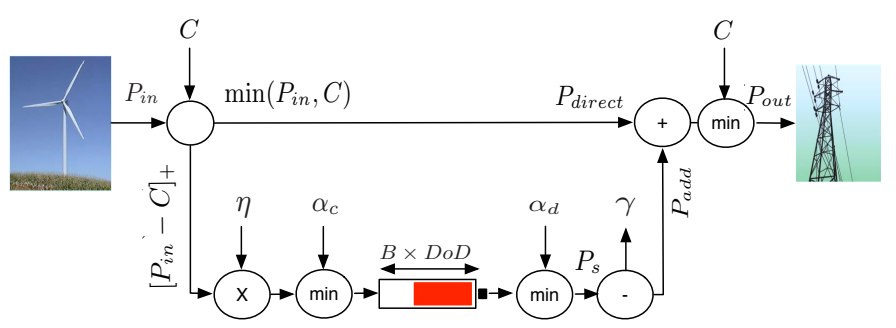

Figure 3: Integrating renewables using storage devices: Using a nonideal storage device with parameters $\left(\alpha_{c}, \alpha_{d}, \eta, D o D, B, \gamma, \lambda\right)$ to transfer renewable power $P_{i n}$ over an access line, with maximum allowable power transmission of $C$.

which is:

$$
R_{0}=\lim _{T \rightarrow \infty} \frac{\sum_{\tau=0}^{T}\left[P_{i n}(\tau)-C\right]_{+}}{\sum_{\tau=0}^{T} P_{i n}(\tau)}
$$

where $[x]_{+}=\max (0, x)$ for any $x$.

With the above notation and definitions, we can now formulate the EROI of a system without storage, as illustrated in Fig. 2. In a system without storage, the only energy invested to create the system is the energy used to produce the energy harvesting technology (wind turbine or solar PV panel). Denote $E R O I_{e}$ as the EROI of the energy harvesting technology for each form of renewable energy (e.g., the EROI of wind turbines or solar PV panels). Then, the EROI of a system without storage depicted in Fig. 2, denoted $E R O I_{0}$, 
is:

$$
E R O I_{0}=\left(1-R_{0}\right) E R O I_{e},
$$

because only a fraction $\left(1-R_{0}\right)$ of the total available energy from the energy harvesting tool is eventually absorbed into the system, due to the access line constraint. Formulating EROI for a system using storage is more complicated and is the focus of the next section.

\subsection{The EROI of a system with storage}

Transmitting renewable energy over an access line using a storage device is depicted in Fig. 3. The available power from the output of the energy harvesting technology at time slot $t$ is $P_{i n}(t)$. The maximum allowable power on the access line is $C$. Thus, $\min \left(P_{i n}, C\right)$ is transmitted directly over the line (without going through the storage device) and the remaining available power (i.e, $\left.\left[P_{i n}(t)-C\right]_{+}\right)$is sent to be stored in the storage device (if possible). The absorbable power at any time comes either directly from the energy harvesting tool, $P_{\text {direct }}$, or from the energy stored in the storage device $P_{a d d}$. Before proceeding with the EROI formulation, we will discuss the non-ideal physical constraints of the storage devices, which influence the analysis.

- Storage size $(B(\mathbf{W h}))$ : This is the maximum amount of energy that can be stored in a storage device.

- Storage charging and discharging rate limit ( $\alpha_{c}$ and $\left.\alpha_{d}(\mathbf{W})\right)$ : This is the limit on the charge or discharge power. Typical discharge rates are many times greater ( $10 \times$ for $\mathrm{PbA}$ and $5 \times$ for lithium-ion batteries) than charging rates.

- Self-discharge $(\gamma(\mathbf{W}))$ : Stored energy leaks over time at rate $\gamma$. Large self-discharge rates become problematic when storage devices need to store large amounts of energy for a long time.

- Efficiency $(0 \leq \eta \leq 1)$ : Due to inherent inefficiencies, each unit of energy stored is reduced to $\eta$ units that can be used later. Storage devices converting electrical energy to other forms typically have lower efficiencies.

- Depth-of-discharge ( $0 \leq$ DoD $\leq 1)$ : While the entire capacity of some storage devices can be used for energy storage, in others, the storage life is extended if only a fraction (DoD) of available capacity is used.

- Cycle life $(\lambda)$ : Let $B$ be the storage size in Watt-hours (Wh) for a storage device, the total energy delivered by that device throughout its lifetime is [17]:

$$
E_{\text {lifetime }}=\lambda \times B \times D_{o} D,
$$

where $\lambda$ is the cycle life of the storage.

A precise state of charge ( $\mathrm{SoC}$ ) analysis must account for the above physical constraints of storage. Although we account for the above physical constraints, there are still some constraints that we do not take into account:
- Temperature: The ambient temperature significantly impacts the storage lifetime; the higher the ambient temperature, the lower the battery lifetime.

We assume that the ambient temperature is not highly variable. This assumption, however, can be simply relaxed and incorporated in our derivations, if the impact of temperature on the storage operation is quantified. This has yet to be modelled in the literature.

- The impact of partial charging/discharging on cycle life: For simplicity, we assume that $\lambda$ in Eq. (9) is a constant for each storage technology. It has, however, been observed that $\lambda$ of some battery technologies is highly affected by the discharge rate, especially during the first few moments of each discharge cycle [12]. This is known as the rate capacity effect. Moreover, it is also a function of the shape of the discharging process. For instance, an intermittent discharging process, which has an on-off duty cycle, leads to a longer lifetime than a steady discharging process. This is known as the recovery effect; during periods with no or very low discharge, the battery can recover the capacity lost during periods of high discharge to a certain extent [8]. Thus, the battery lifetime can be improved by using a pulsed current discharge instead of a constant current discharge, due to the recovery effect.

The discharging process of storage systems, when used to back up renewable energy sources, is characterized by highly variable charging power, deep cycling, partial cycling, and infrequent full charging. It has been shown in [14], that although the variability in renewable energy (wind) dramatically decreases the lifetime of $\mathrm{PbA}$ batteries, it does not considerably impact the lifetime of lithium-ion batteries. Thus, in our numerical examples, the EROI values of lithium-ion are close to practical values for renewables, while those corresponding to $\mathrm{PbA}$ might overestimate the practical values. This is not problematic, however, as the EROI values of $\mathrm{PbA}$ in our examples are already below the values of a system without storage.

In the next section, we formulate the EROI, assuming all of the storage imperfections depicted in Fig. 3.

\subsection{SoC analysis}

Due to the limited access line capacity in the scenario depicted in Fig. 3, the instantaneous surplus power at any time $t,\left[P_{i n}(t)-C\right]_{+}$, is sent to be stored in the storage device. However, because of the non-ideal behaviour of the storage devices mentioned earlier, part of this power will be wasted and cannot be withdrawn later. The maximum charging power of the storage cannot exceed a certain threshold $\alpha_{c}$ and any charging power beyond that, is curtailed. Moreover, the storage device loses a fraction $1-\eta$ of the total energy being stored in the storage device, due to storage inefficiency. Energy stored in the storage device is discarded 
with rate $\gamma$, due to storage self-discharge. The storage cannot be discharged faster than $\alpha_{d}$. The storage lifetime constraint is met if only a DoD fraction $\leq 1$ of the entire storage is used. Thus, at any moment, the storage state of the charge cannot exceed $D o D \times B$. Finally, at any time $t$ due to the constraint of the line capacity, only up to a maximum of $\left[C-P_{i n}(t)\right]_{+}$ can be sent to the line from the storage.

Accounting for all of the above parameters, we can formulate the state of charge $b(t)$ of the storage at any time instant $t$. The storage device in such a system can be viewed as a buffer. Similar to a buffer content formulation, a state of charge formulation can be expressed by a recursive equation as follows:

$$
\begin{aligned}
b(t)=\min & \left(B \times \operatorname{DoD},\left[\min \left(\left[P_{i n}(t)-C\right]_{+}, \alpha_{c}\right) \eta\right.\right. \\
& \left.\left.-\min \left(\left[C-P_{i n}(t)\right]_{+}, \alpha_{d}\right)-\gamma+b(t-1)\right]_{+}\right) .
\end{aligned}
$$

To simplify notation, we define $b^{\prime}(t)$ to be the state of charge at time $t$ before deducting the storage output processes $\left(P_{s}\right.$ in the system model in Fig. 3), i.e.,:

$$
\begin{array}{r}
b^{\prime}(t)=\min \left(B \times D o D,\left[\min \left(\left[P_{\text {in }}(t)-C\right]_{+}, \alpha_{c}\right) \eta\right.\right. \\
\left.+b(t-1)]_{+}\right) .
\end{array}
$$

Then, the output process from the storage device is:

$$
P_{s}(t)=\min \left(\min \left(\left[C-P_{i n}(t)\right]_{+}, \alpha_{d}\right)+\gamma, b^{\prime}(t)\right)
$$

and the additional power being transmitted over the line with respect to the no-storage scenario is:

$$
P_{a d d}(t)=\left[P_{s}(t)-\gamma\right]_{+} .
$$

Let $E_{i n}(T)$ be the total available energy from the energy harvesting device in the interval $[0, T]$ for some $T>0$, computed from Eq. (4). We also define $E_{\text {direct }}(T), E_{a d d}(T)$, and $E_{s}(T)$, respectively, as the total energy directly transported from the renewable energy source, the total energy coming from the storage device, and the total energy withdrawn from the storage device in the time interval $[0, T]$. These quantities are, respectively, given by

$$
\begin{aligned}
& E_{\text {direct }}(T)=\sum_{\tau=0}^{T} \min \left(P_{i n}(\tau), C\right), \\
& E_{\text {add }}(T)=\sum_{\tau=0}^{T} P_{a d d}(\tau),
\end{aligned}
$$

and

$$
E_{s}(T)=\sum_{\tau=0}^{T} P_{s}(\tau)
$$

Using Eqs. (4)-(14) together with these new definitions and notation, the waste ratio in a system without storage can be rewritten as:

$$
R_{0}=1-\lim _{T \rightarrow \infty} \frac{E_{\text {direct }}(T)}{E_{\text {in }}(T)} .
$$

Moreover, the waste ratio (defined in Eq. (6)) in a system with storage is:

$$
R_{B}=R_{0}-\lim _{T \rightarrow \infty} \frac{E_{a d d}(T)}{E_{\text {in }}(T)} .
$$

There are two devices used in the scenario depicted in Fig. 3: the energy harvesting technology and the energy storage with their respective EROI denoted as $E R O I_{e}$ and $E R O I_{s}$. The energy invested to produce a storage system is usually expressed in terms of energy invested to create one unit of that storage system $\varepsilon_{s}$. The $E R O I_{s}$ of a storage system can be subsequently computed as

$$
\begin{aligned}
E R O I_{s} & =\frac{\eta E_{\text {lifetime }}}{B \times \varepsilon_{s}} \\
& =\frac{D o D \times \lambda \eta}{\varepsilon_{s}},
\end{aligned}
$$

where Eq. (9) is used to obtain the second line.

The energy return in the time interval $[0, T]$ in this model is:

$$
\text { Energy return in }[0, T]=E_{\text {direct }}(T)+E_{a d d}(T),
$$

and the energy invested in the time interval $[0, T]$ is:

$$
\text { Energy invested in }[0, T]=\frac{E_{i n}(T)}{E R O I_{e}}+\frac{E_{s}(T)}{E R O I_{s}} \text {. }
$$

Note that Eqs. (20) and (21) compute the exact energy return and energy investment in $[0, T]$ based on the actual energy flux in that period. This models the fact that the lower the amount of energy generated by the source, the longer its lifetime. Similarly if there is zero energy flux through the storage element in that period, there is no degradation in its expected lifetime. Although this is a simplifying assumption, it does reflect the fact that the lifetime of a generator or storage element is coupled to the energy flux it generates, stores, or releases.

Combining Eq. (20)-(21), we have:

$$
\begin{aligned}
E R O I_{B} & =\lim _{T \rightarrow \infty} \frac{E_{\text {direct }}(T)+E_{a d d}(T)}{\frac{E_{\text {in }}(T)}{E R O I_{e}}+\frac{E_{s}(T)}{E R O I_{s}}} \\
& =\lim _{T \rightarrow \infty} \frac{\left(1-R_{0}\right)+E_{a d d}(T) / E_{i n}(T)}{\frac{1}{E R O I_{e}}+\frac{E_{s}(T)}{E R O I_{s} \times E_{i n}(T)}},
\end{aligned}
$$

where we have used Eq. (17) in the second line.

We learn from Eqs. (8)-(23) that the cycle life of a storage technology must be larger than a critical life cycle $\lambda_{c r}$, to be beneficial (with respect to curtailment) to be used for the access line, where:

$$
\lambda_{c r}=\lim _{T \rightarrow \infty} \frac{\left(1-R_{0}\right) \varepsilon_{s} \times E R O I_{e} \times E_{s}(T)}{\eta \times D o D \times E_{a d d}(T)} .
$$

Otherwise, curtailment has a larger EROI than a system with storage, and hence, is more energy efficient. Comparing the 


\begin{tabular}{|c||c|c|c|}
\hline & PbA & Lithium-ion & CAES \\
\hline \hline Efficiency & 0.75 & 0.85 & 0.68 \\
\hline Charge time (=storage size/charge rate) & $8-16 \mathrm{~h}$ & $2-4 \mathrm{~h}$ & $15 \mathrm{~min}$ \\
\hline Discharge rate to charge rate ratio & 10 & 5 & 4 \\
\hline Self-discharge/day (=self-discharge rate $\times$ day/storage size) $(\%)$ & 0.3 & 0.1 & 0 \\
\hline DoD & 0.80 & 0.80 & 1 \\
\hline$\varepsilon_{s}$ & 96 & 136 & 22 \\
\hline$\lambda$ & 700 & 6000 & 25000 \\
\hline
\end{tabular}

Table 3: Characteristics of storage technologies [1], [6], [22]

current life cycle of the storage technologies with $\lambda_{c r}$ determines if those existing storage technologies outperform curtailment for integrating renewables.

\section{NUMERICAL EXAMPLES}

In this section, we numerically evaluate the energy efficiency of adding storage to an access line that is used to carry renewable power by comparing the EROI of systems with and without storage. For wind power, we use the measurement traces collected from wind turbines located on the west coast of the United States, with 10-minute time resolution $\left(T_{u}=10 \mathrm{~min}\right)$, freely available in [2]. For solar power, we use the solar irradiance dataset from the atmospheric radiation measurement (ARM) website [3] from the $C 1$ station in southern great plains (SGP) permanent site with a 1-minute time resolution. We multiply the solar irradiance by a PV efficiency factor of 0.2 to translate from solar irradiance to the power output of a solar PV panel of unit size $\left(1 \mathrm{~m}^{2}\right)$. To obtain the output power of a solar PV farm, the output power of a unit size PV panel is multiplied by the total surface area of all of the existing solar PV panels in that farm.

We consider three storage technologies in our examples:

1. Lead-acid battery (PbA): This storage technology is widely-used, due to its low price, simple manufacturing, and lowest self-discharging rate among all batteries. It has, however, a small charging rate, and a limited life cycle.

2. Lithium-ion battery (Li-ion): This type of battery has a low discharge rate and a larger-than-average cycle life, compared to other batteries. A disadvantage is its price, which can be three times that of a PbA battery.

3. Compressed air energy storage (CAES): This device stores energy in the form of compressed air and releases it to rotate a turbine for electricity generation. CAES has a low efficiency, but has no restriction on $\mathrm{DoD}$, and a large cycle life.

The characteristics of these technologies are given in Table 3 . In our examples, we define $P_{\max }$ to be the peak generation capacity of the renewable energy farm, i.e.,:

$$
P_{\max }=\max _{t \geq 0}\left(P_{i n}(t)\right) \text {. }
$$

Our example solar and wind power farms both have $P_{\max }=$ $3 \mathrm{MW}^{2}$. We treat the normalized access capacity, defined as $C / P_{\max }$, as the independent variable. This indicates how large the line capacity is with respect to the maximum renewable power.

First, we study the EROI of different storage technologies as a function of normalized access capacity (Section 5.1). We then study how to size access lines to achieve maximum EROI (Section 5.2). Finally, we study the improvements that need to be made in storage cycle life to allow the EROI to at least match that of curtailment (Section 5.3).

\subsection{The EROI of storage technologies}

In this section, we study whether adding storage increases the EROI of the system compared to curtailment (Fig. 2). We use Eq. (23) to compute the EROI of a system with storage $\left(E R O I_{B}\right)$ with $B=10 \mathrm{MWh}$, accounting for all physical constraints of storage systems. We also include the EROI of an ideal storage technology (using Eq. (23) with $B=\infty$, $\alpha_{c}, \alpha_{d}=\infty, \gamma=0$, but with the same $\lambda$ ) and the EROI of a system without storage from Eq. (8) $\left(E R O I_{0}\right)$. Adding storage to the system is beneficial if it improves the EROI of the system with respect to curtailment, i.e., $E R O I_{B}>$ $\mathrm{EROI}_{0}$.

Fig. 4 shows that the EROI impact of adding storage depends on the storage technology, the type of renewable energy (e.g., solar or wind power), and the normalized access capacity $C / P_{\max }$. In particular, we find that the choice of storage technology determines the EROI gain over curtailment ('No storage'); using some storage technologies is beneficial (such as lithium-ion and CAES), while for some other storage technologies (such as $\mathrm{PbA}$ ), it is more energy efficient to curtail the surplus power.

Comparing the non-ideal and ideal curves in Fig. 4a for wind power shows that considering non-ideal behaviour of the storage technologies substantially affects the EROI. In other words, an idealized analysis might significantly overestimate or underestimate the EROI, possibly yielding misleading conclusions. In contrast, for the storage size chosen here, the ideal analysis for solar power closely matches that for non-ideal storage. This is because this storage size ( $B=10 \mathrm{MWh}$ ) is sufficiently large so that it behaves as an infinite size storage. We study the effect of smaller storage

\footnotetext{
${ }^{2}$ We choose the surface area of the total PV panels in the solar PV farm such that $P_{\max }=3$ with the given solar irradiance dataset.
} 


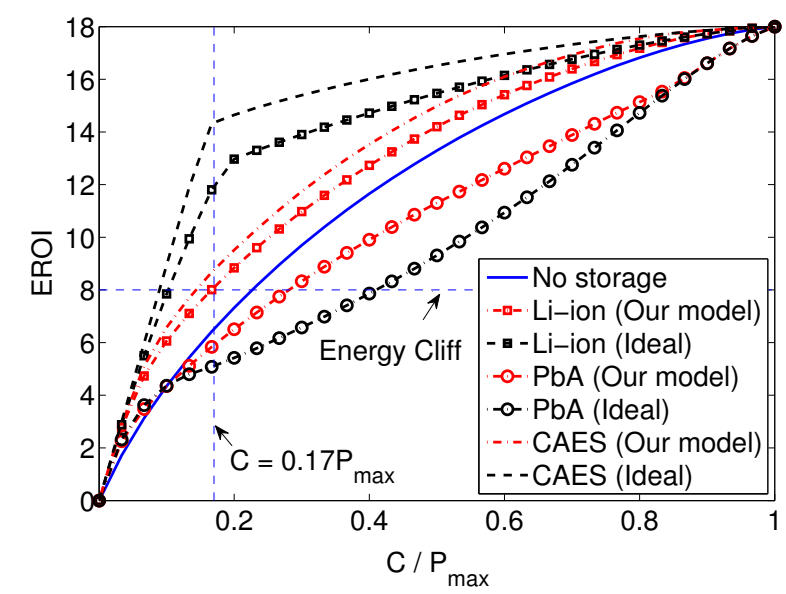

(a) Wind

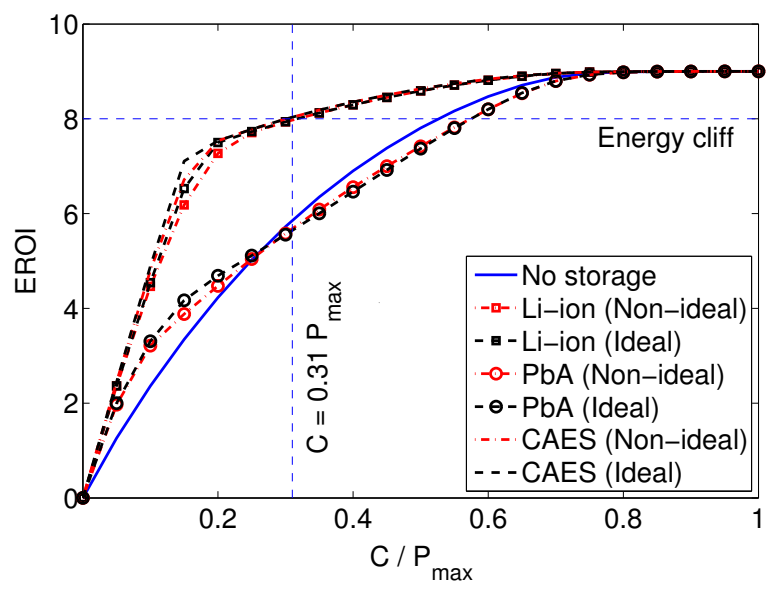

(b) Solar

Figure 4: Energy evaluation of storage systems and comparison with no-storage and ideal storage models: EROI as a function of the normalized access capacity. The storage sizes of non-ideal models are $\mathrm{B}=10 \mathrm{MWh}$.

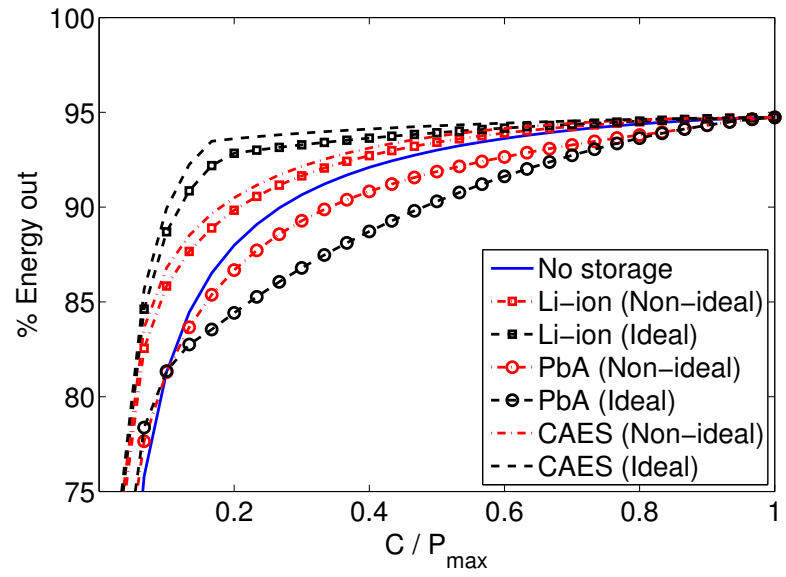

(a) Wind

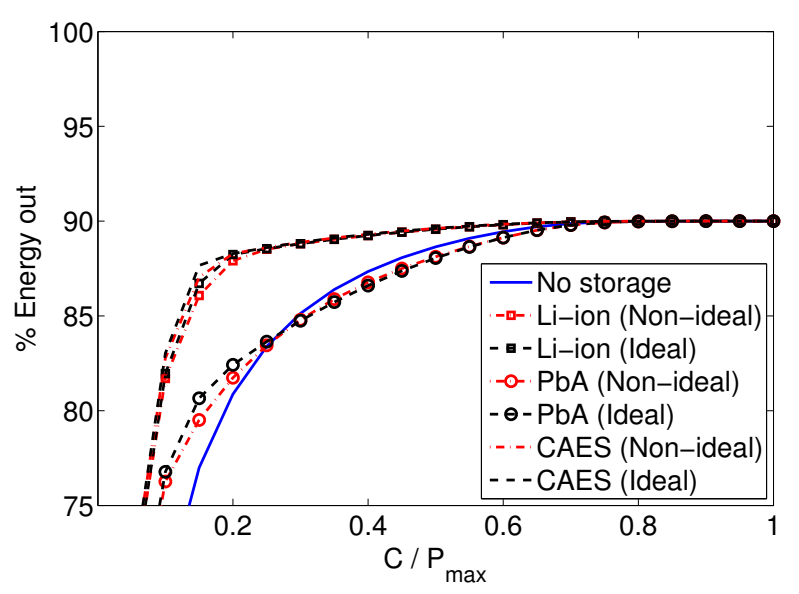

(b) Solar

Figure 5: Illustrating the energy cliff using our analysis and the comparison with no-storage and ideal storage models: Output energy percentage as a function of the normalized access capacity. The storage sizes of non-ideal models are $\mathrm{B}=10 \mathrm{MWh}$. Note that the $\mathrm{Y}$ axis does not start at the origin.

size in Fig. 6. Surprisingly, we find that the EROI of an ideal store can be smaller than that of a non-ideal one (e.g., for $\mathrm{PbA}$ batteries). This counter intuitive observation occurs because an ideal storage device carries more energy flux compared to a non-ideal one in the same finite time interval; hence, it increases the energy investment in that interval.

We have drawn a horizontal line at EROI $=8$ in Fig. 4 , which corresponds to the critical net energy cliff (see Section 2). As discussed in Section 2, we prefer to design energy systems that have an EROI $\geq 8$. The intersection of the line EROI $=8$ and the EROI curves determine the access capacity needed to guarantee that the system operates above the net energy cliff. The access line capacity required to satisfy this constraint depends on the storage technology, storage size, and the type of renewable source. Access lines are expensive and the cost increases with both the length of the line and with its capacity [21]. Thus, the minimum access line capacity that can guarantee a quality constraint (such as $E R O I>8)$ is desirable. A comparison of the intersecting points for a non-ideal and ideal storage model suggests that an ideal storage model can be misleading, if it is used to estimate the EROI of a system with storage. Moreover, a comparison of these intersecting points for wind and solar power reveals that integrating solar power is much more expensive than integrating wind power. For instance, the required access line capacity for solar power with lithium-ion storage is $C=0.31 P_{\max }$ which is almost twice as much as that for wind power $C=0.17 P_{\max }$ (recall that $P_{\max }$ is equal for both solar and wind power in our examples). This is due to the larger variance of solar power compared to wind power. 


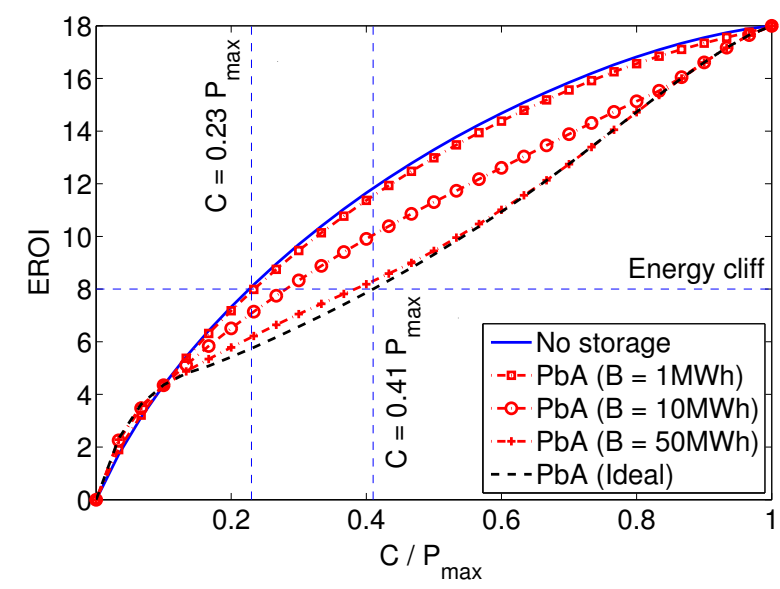

(a) Wind (PbA storage)

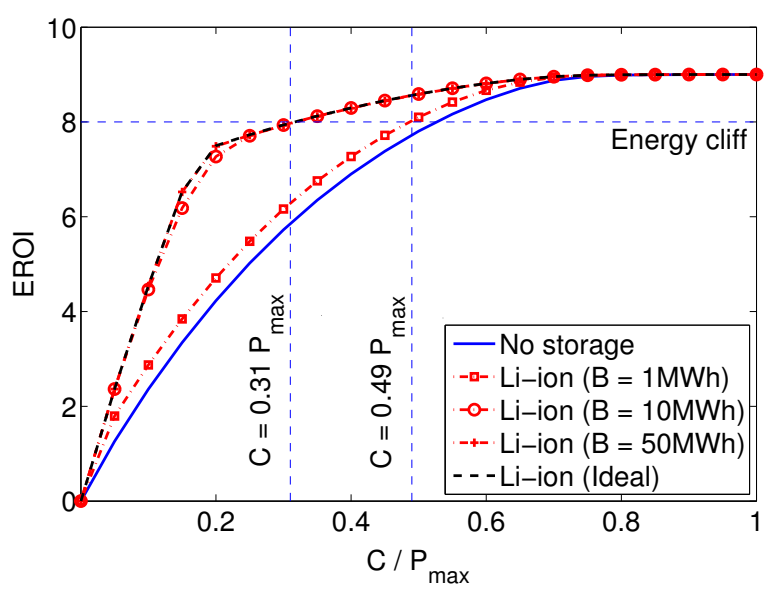

(b) Solar (Lithium-ion storage)

Figure 6: EROI as a function of storage size and normalized access capacity for both ideal and non-ideal storage for specific storage technologies.

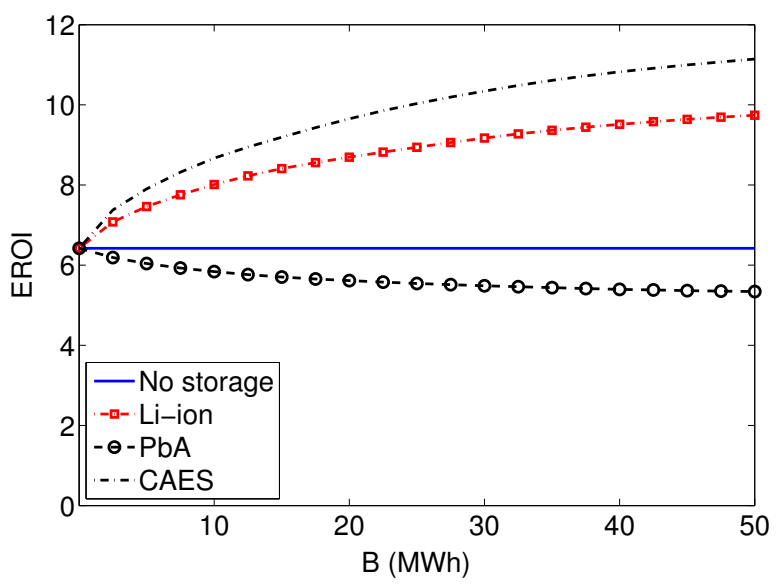

(a) Wind $\left(C / P_{\max }=0.17\right)$

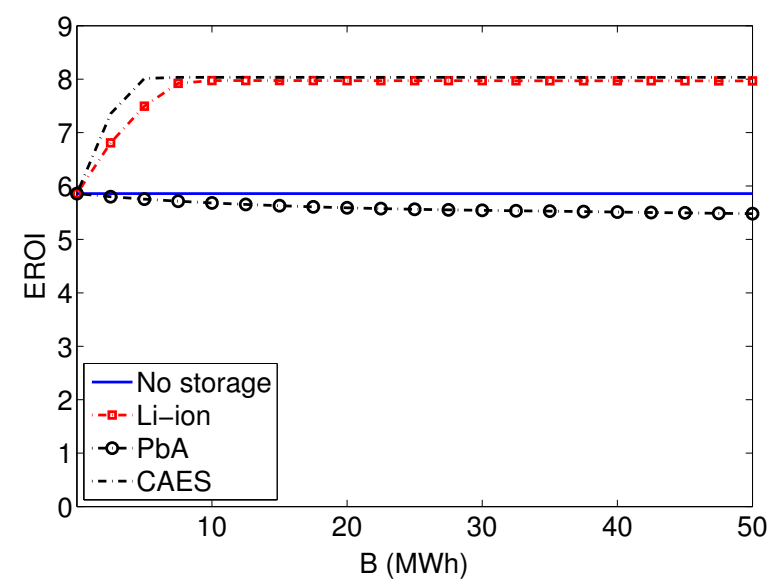

(b) Solar $\left(C / P_{\max }=0.31\right)$

Figure 7: The impact of storage size on EROI: EROI as a function of storage size.

Specifically, there is an underlying diurnal pattern for solar power which dominates its short-term variations. However, wind power lacks such a deterministic pattern and its variations are nearly unbiased. Thus, for the same $P_{\max }$ and $C$, solar power has a larger curtailment ratio for solar power than wind power.

Although the storage size is assumed to be $10 \mathrm{MWh}$ in this example, all scenarios with storage coincide with that of a system with no storage when $C / P_{\max }=1$. This is due to the fact that when $C / P_{\max }=1$, the net energy flux through the storage element is zero, thus i has no effect on EROI.

The impact of increasing the access line capacity to avoid the net energy cliff is illustrated in another way in Fig. 5. This graph repeats the example in Fig. 4, however, it shows the percentage of energy return to the total energy (i.e., $\frac{E R O I}{E R O I+1}$ ). This graph shows that the energy cliff occurs at different line access capacities, depending on the storage technologies and types of renewable energy sources. Moreover, this figure confirms the need to account for the non-ideal behaviour of storage devices.

\subsection{The effect of storage size on the EROI}

Fig. 6 shows EROI analysis with both ideal and non-ideal storage for specific storage technologies associated with wind and solar power. It is evident that EROI analysis using idealized storage assumptions can be misleading. Indeed, the difference is more pronounced for smaller storage sizes (note that with idealized storage assumptions we have $B=\infty$ ). For example, from Fig. 6a, the points corresponding to the net energy cliff (i.e., EROI $=8$ ) are $C / P_{\max }=0.23$ and $C / P_{\max }=0.41$, respectively, for a PbA battery with size $B=1 \mathrm{MWh}$ and for an ideal storage model. The corre- 


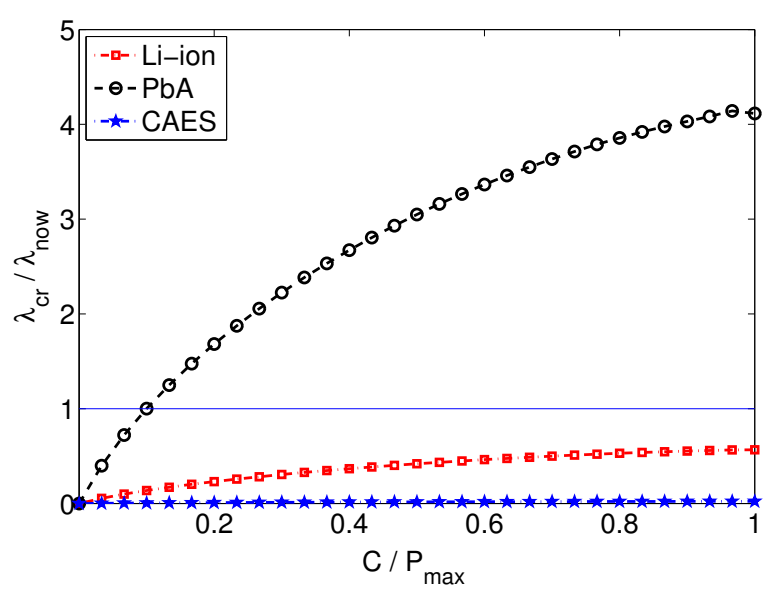

(a) Wind

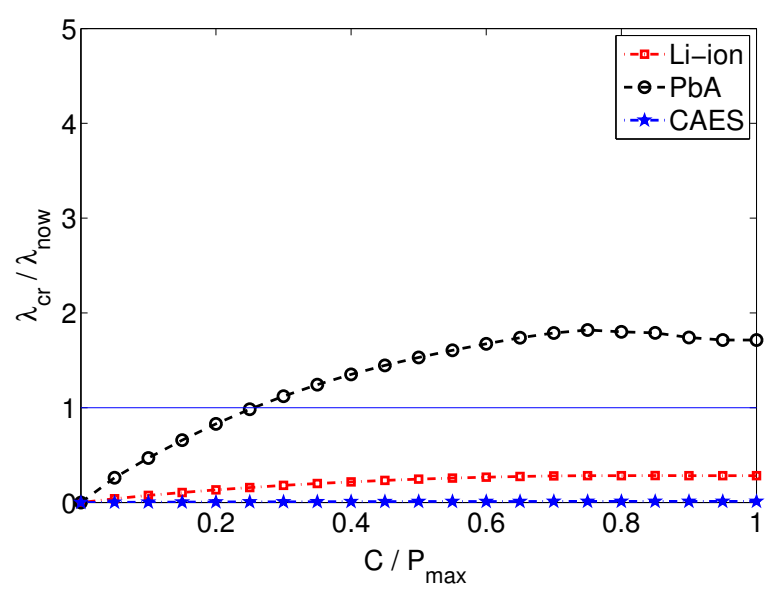

(b) Solar

Figure 8: The ratio of critical cycle life to current cycle life for different storage technologies with $B=10 \mathrm{MWh}$ : The cycle life must be scaled by these values for the storage devices so that they are sufficiently energy efficient to replace curtailment.

sponding points in Fig. $6 \mathrm{~b}$ also differ considerably, with $C / P_{\max }$ $=0.49$ for a non-ideal storage model and $C / P_{\max }=0.31$ for that with idealized storage assumptions.

To demonstrate that increasing the storage size can have either a positive or negative impact on EROI, in Fig. 6 we show the $E R O I_{B}$ for three different storage sizes $(B=$ $1,10,50 \mathrm{MWh}$ ) of $\mathrm{PbA}$ for wind power and lithium-ion for solar power. Indeed, if $E R O I_{B}<E R O I_{0}$ for $B>0$, then $E R O I_{B}$ is increasing in $B$. However, if $E R O I_{B}>$ $E R O I_{0}$ for $B>0$, then $E R O I_{0}$ is decreasing in $B$.

Unlike the simple model analysis in [6], which is oblivious to the choice of storage size, our analysis takes the storage size into account. Hence, this allows storage sizing for a target performance. Specifically, Fig. 7 illustrates the impact of increasing the storage size from another perspective. In this graph, for a fixed operation point of $C / P_{\max }=0.31$ for solar power and $C / P_{\max }=0.17$ for wind power, we compute $E R O I^{3}$. At $B=0$, the curtailment and storage systems are equivalent. As $B$ increases, the EROI monotonically converges to a system with the same storage technology with infinite size. The speed of convergence depends on the storage technology, type of renewable energy, and point of operation. If $E R O I_{B}<E R O I_{0}$ for $B>0$, then the optimal storage size is zero. If $E R O I_{B}>E R O I_{0}$ for $B>0$, then the optimum storage size is the minimum storage size which achieves an EROI satisfactorily close to the EROI of an infinite storage size with the same storage technology. The monotonic trend observed in Fig. 7 is attributed to the fact that the storage size only limits the amount of power that can be absorbed or discharged, but it does not directly impact EROI analysis. Indeed, the EROI of an ideal storage device is independent of $B$.

\footnotetext{
${ }^{3}$ These are the corresponding operating points at which the systems with lithium-ion batteries in Fig. 4 reach the energy cliff (EROI = 8).
}

\subsection{Critical cycle life for a storage technology}

Following [6], we now study the critical cycle life, which is defined as the cycle life for which the EROI of a system with storage is equal to that of a system with no storage. This is denoted as $\lambda_{c r}$ in Eq. (24). The critical cycle life is helpful in assessing the improvements necessary to make a storage technology viable.

Fig. 8 illustrates the ratio of the critical cycle life to the current cycle life for existing technologies (i.e., $\lambda_{c r} / \lambda_{\text {now }}$ ) as a function of normalized access capacity. If this ratio is less than unity for a storage technology, then using this storage increases EROI compared to the curtailment scenario. Otherwise, that storage technology is not preferred to curtailment, and future improvements need to scale its cycle life by $\lambda_{c r} / \lambda_{\text {now }}$. As Fig. 8 shows, with the existing technologies, it is efficient to use lithium-ion batteries and CAES for both wind and solar power. However, $\mathrm{PbA}$ batteries are not yet efficient enough to compete with pure curtailment.

As shown in Fig. 8, the critical cycle life mostly increases as the access line capacity increases. This is because the critical cycle life from Eq. (24) is proportional to $\left(1-R_{0}\right)$, and this term usually increases as the access capacity increases. The physical interpretation is that as the access capacity increases, the waste ratio decreases, thus, there is less power to be saved by storage. Thus, a storage technology must have a larger cycle life to increase EROI beyond curtailment for large values of $C / P_{\max }$. Note that this ratio does not always increase with access line capacity; there is also a small decrease in $\lambda_{c r}$ when $C / P_{\text {max }}$ increases for the case of PbA. It can be shown that this is due to the high self-discharge rate of this technology.

\section{RELATED WORK}

Cost efficiency is a significant-if not the only-factor that 


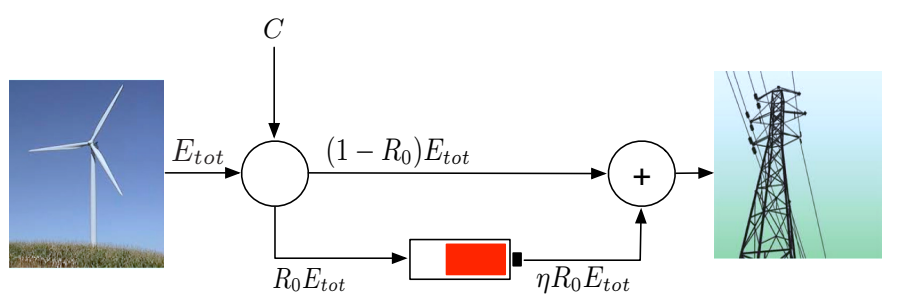

Figure 9: Simple model used in [6]: Idealized storage assumption ( $B=$ $\infty, \alpha_{c}, \alpha_{d}=\infty$, and $\gamma=0$ ) and no access capacity constraint on the power transmitted from the storage.

has been studied in the literature to decide whether to add storage to an energy system. The fundamental problem studied in these cases is whether the additional revenue obtained from an energy system by adding storage is larger than the investment cost to buy that storage. The results of such a study are highly dependent on the use to which the energy system is put to, as well as the storage technology. For example, it is shown in [11] that adding Sodium-Sulphur batteries at current costs increases the revenue for RE farms. In another work [5], a cost/benefit analysis was conducted by comparing the annual cost of different storage technologies with the total profit for both the utility and an RE farm owner. The results show that integrating storage with the distribution system is economically feasible only when the least expensive storage ( $\mathrm{Zn}-\mathrm{Br})$ is used.

Evaluating the energy efficiency of an energy system using EROI analysis has drawn much attention over the last few years. Prior studies are mostly focused on the following five topics [18]: (1) energy efficiency of corn ethanol; (2) comparing the EROI of major fuel types; (3) energy return on water invested (EROWI); (4) the relation between EROI and the economy; and (5) an attempt to calculate the minimum EROI for a sustainable society. None of these are directly related to our work.

Evaluating the energy efficiency of adding storage to RE farms was recently proposed by Barnhart et al [6]. This work introduced the fresh, new perspective of EROI analysis to study this important problem. Using a simple model of an RE farm (see Fig. 9) and approximate analysis, their work showed that, in contrast to conventional wisdom, the EROI of an RE farm sometimes decreases by adding storage.

In their model, if $E_{t o t}$ represents the total available energy from an RE harvesting technology (such as wind turbine or photovoltaic) over a long time interval, then only a fraction $\left(1-R_{0}\right)$ of that total energy can be transmitted over an access line. The rest of the energy is stored in the storage device and $R_{0} \eta$ portion of that is withdrawn later, where $\eta$ is the efficiency of that storage system. This model has several shortcomings:

First, it makes strong assumptions on storage: infinite storage capacity, infinite charge and discharge rates, and zero self-discharge. Second, it permits infinite discharge rate from the storage device into the access line. Third, it treats, $R_{0}$, the waste of power ratio with no storage, as an independent variable, rather than an outcome of the underlying storage charge/discharge process. Not only does this disregard the dynamics of the system but also, given that the waste ratio in a system with storage is smaller than $R_{0}$ (compare Eq. (7) and Eq. (18)), it is technically incorrect to compare $E R O I_{0}$ and $E R O I_{B}$ for the same $R_{0}$ in the first place.

How important are these shortcomings? Figs. 4, 5, and 6 show that even when we consider only one of the inaccuracies in the simple model-assuming an ideal store- the resulting EROI considerably differs from that computed from a more complete analysis. This suggests that the conclusions in [6] may have substantial shortcomings. For instance, this work claims that using any type of storage device for solar power always increases EROI and that using batteries for wind power always decreases EROI. Our results contradict these conclusions, as discussed next.

\section{CONCLUSIONS AND FUTURE WORK}

Integrating RE farms into the power grid is challenging due to inherent unpredictable fluctuations. One of the problems arising in energy integration is that the peak power bursts cannot be absorbed to the grid due to the limited access line capacity. A common solution to this problem is to simply curtail excess power. Curtailment is simple and does not need any additional hardware; however, it leads to significant power waste. A promising solution to reduce the waste ratio is to use storage devices to store some of the curtailed power. Recent work suggests that to fairly evaluate the energy benefits of adding storage to a system, the energy invested to create the storage device must also be taken into account [6]. This can be achieved by using a well-known metric called energy return on investment (EROI); adding a storage device to an energy system is energy efficient only if it improves the EROI of that system.

In this work, we present an accurate EROI analysis for RE farms. This analysis accounts for several storage imperfections and tracks the dynamics of the state of charge process. Other than illustrating why imperfections of a storage system must be accounted for, our analysis when applied to actual solar and wind power measurement traces leads to several new insights:

- The required access line capacity to achieve a certain EROI is much larger for solar power than for wind power. This implies that solar power integration is more expensive than wind power.

- EROI always monotonically varies as a function of storage size; it could, however, be either increasing or decreasing.

- Some existing technologies, such as lithium-ion batteries and CAES increase the EROI in comparison with curtailment whereas some others such as $\mathrm{PbA}$ have a lower EROI than curtailment. 
- We contradict earlier claims in [6] that no battery technology is energy efficient for wind and all battery technologies are energy efficient for solar power.

We caution that these insights might be sensitive to the numerical values in our analysis.

In future work, we plan to use the insights gained here to tackle the complex problem of EROI analysis of RE farms whose output is curtailed due to limited demand.

\section{REFERENCES}

[1] http: / /batteryuniversity.com.

[2] http://wind.nrel.gov/Webnrel/.

[3] http: //www . archive.arm.gov.

[4] E. W. E. Association. Wind energy-the facts: a guide to the technology, economics and future of wind power. Earthscan, 2009.

[5] Y. M. Atwa and E. F. El-Saadany. Optimal allocation of ESS in distribution systems with a high penetration of wind energy. IEEE Transactions on Power Systems, 25(4): 1815 - 1822, 2010.

[6] C. J. Barnhart, M. Dale, A. R. Brandt, and S. M. Benson. The energetic implications of curtailing versus storing solar- and wind-generated electricity. Energy Environment Science, 6:2804 - 2810, 2013.

[7] A. Carvallo and J. Cooper. The Advanced Smart Grid: Edge Power Driving Sustainability. Artech House, 2011.

[8] C. Chiasserini and R. R. Rao. A model for battery pulsed discharge with recovery effect. In Proc. of IEEE WCNC, pages 636-639, 1999.

[9] R. A. F. Currie, G. W. Ault, and J. R. McDonald. Methodology for determination of economic connection capacity for renewable generator connections to distribution networks optimised by active power flow management. IEE ProceedingsGeneration, Transmission and Distribution, 153(4):456 - 462, 2006.

[10] P. Denholm. Energy storage to reduce renewable energy curtailment. In IEEE Power and Energy Society General Meeting, pages 1 - 4, 2012.

[11] S. Gill, G. W. Ault, and I. Kockar. The optimal operation of energy storage in a wind power curtailment scheme. In Proc. of IEEE Power and Energy Society General Meeting, pages 1- 8, 2012.

[12] J. Groot. State-of-Health Estimation of Li-ion Batteries: Cycle Life Test Methods. PhD thesis, Chalmers University of technology, 2012.

[13] C. A. S. Hall, S. Balogh, and D. J. R. M. What is the minimum eroi that a sustainable society must have? Energies, 2(1):25 - 47, 2009.

[14] E. M. Krieger. Effects of variability and rate on battery charge storage and lifespan. $\mathrm{PhD}$ thesis, Princeton University, 2013.

[15] J. Lambert, J. Hall, S. Balogh, A. Poisson, and A. Gupta. EROI of global energy resources preliminary status and trends. Technical report, State University of New York, College of Environmental Science and Forestry, November 2012.

[16] S. N. Liew and G. Strbac. Maximising penetration of wind generation in existing distribution networks. IEE Proceedings-Generation, Transmission and Distribution, 149(3):256 - 262, 2002.

[17] N. Michelusi, L. Badia, R. Carli, L. Corradini, and M. Zorzi. Impact of battery degradation on optimal management policies of harvesting-based wireless sensor devices. In Proc. of IEEE INFOCOM, pages 590 - 594, 2013.

[18] D. J. Murphy and C. A. S. Hall. Year in review: EROI or energy return on (energy) invested. Annals of the New York Academy of Sciences, 1185(1):102 - 118, 2010.

[19] L. F. Ochoa, C. J. Dent, and G. P. Harrison. Distribution network capacity assessment: Variable DG and active networks. IEEE Transactions on Power Systems, 25(1):87 - 95, 2010.

[20] J. Rogers, S. Fink, and K. Porter. Examples of wind energy curtailment practices. Technical Report SR-550-48737, National Renewable Energy Library, July 2010.

[21] S. Stoft, C. Webber, and R. H. Wiser. Transmission pricing and renewables: Issues, options, and recommendations. Technical Report LBNL-39845, LBNL, May 1997.

[22] D. Wang, C. Ren, A. Sivasubramaniam, B. Urgaonkar, and H. Fathy. Energy storage in datacenters: what, where, and how much? In Proc. of ACM SIGMETRICS/PERFORMANCE, pages 187-198, June 2012. 